\title{
STUDY OF HYPONATREMIA IN PAEDIATRIC PATIENTS WITH ACUTE LOWER RESPIRATORY TRACT INFECTION
}

\author{
Shefali Shashank Mhatre1, Suhas G. Kumbhar ${ }^{2}$ \\ 1IIIrd Year Junior Resident, Department of Paediatrics, Bharati Vidyapeeth (Deemed to be University) Medical College and Hospital, \\ Sangli, Maharashtra, India. \\ 2Professor and P. G. Guide, Department of Paediatrics, Bharati Vidyapeeth (Deemed to be University) Medical College and Hospital, \\ Sangli, Maharashtra, India.
}

\begin{tabular}{l}
\hline ABSTRACT \\
BACKGROUND \\
Hyponatremia is one of the most common electrolyte abnormalities seen in paediatric intensive care unit. Common conditions \\
associated with hyponatremia are pneumonia, acute meningitis, and Kawasaki disease. We wanted to estimate the frequency of \\
hyponatremia and severity of hyponatremia with pneumonia grading. We also wanted to compare the occurrence of hyponatremia \\
in pneumonia and other types of lower respiratory tract infection.
\end{tabular}

\section{METHODS}

This is a hospital based cross-sectional study of children aged 2 months to 5 years suffering from acute lower respiratory tract infection, admitted in PICU or paediatric ward.

\section{RESULTS}

85 cases of lower respiratory tract infection (8\%) of a total of 925 admissions during study period were included in the study. Out of the 85 cases of lower respiratory tract infection, $59(69.4 \%)$ had pneumonia, $16(18.8 \%)$ had bronchiolitis, $8(9.4 \%)$ had wheeze associated lower respiratory tract infection and $2(2.35 \%)$ presented with acute croup syndrome. The prevalence of hyponatremia in lower respiratory tract infection was found to be $42.3 \%$. Out of 36 cases of hyponatremia associated with lower respiratory tract infection, 25 (69.4\%) had mild hyponatremia, 8 (22.2\%) had moderate hyponatremia and 3 (8.3\%) had severe hyponatremia. In our study, out of 36 cases of hyponatremia, 33 (91.66\%) were present in pneumonia group and only 3 (8.33\%) presented in nonpneumonia group. $\mathrm{p}$ value was 0.00 and $\mathrm{z}$ value is 3.817 . The results were statistically significant. Hyponatremia was found in all cases of very severe pneumonia i.e. $13(100 \%)$ as compared to $16(53.33 \%)$ in severe pneumonia group and only $3(18.75 \%)$ in pneumonia group, but there was no significant association between severity of hyponatremia and pneumonia grading as the $\mathrm{p}$ value was $0.145(<0.05)$.

\section{CONCLUSIONS}

The prevalence of hyponatremia in paediatric patients with lower respiratory tract infection was found to be $42.3 \%$. Hyponatremia was more common in pneumonia group (91.66\%) as compared to the non-pneumonia group (8.33\%). Severe hyponatremia was seen more commonly with increase in severity of pneumonia; so diagnosing hyponatremia in early stage and correcting by appropriate interventions plays an important role in outcome of children suffering from pneumonia.

HOW TO CITE THIS ARTICLE: Mhatre SS, Kumbhar SG. Study of hyponatremia in paediatric patients with acute lower respiratory tract infection. J. Evolution Med. Dent. Sci. 2019;8(21):1704-1707, DOI: 10.14260/jemds/2019/375

\section{BACKGROUND}

Lower respiratory tract infection (LRTI) is an important health problem seen in children below 5 years of age needing hospitalisation and attributes to $30 \%$ of deaths yearly worldwide, especially due to pneumonia.(1) Lower respiratory tract infection is an infection that occurs below the level of vocal cord and includes: Bronchitis, tracheitis, bronchiolitis, severe acute respiratory syndrome (SARS), wheeze associated lower respiratory tract infection, pneumonia and empyema. It is inflammation of the airway or pulmonary tissue, due to viral or bacterial infection, below the level of larynx. $(2,3)$

'Financial or Other Competing Interest': None.

Submission 08-04-2019, Peer Review 14-05-2019,

Acceptance 20-05-2019, Published 27-05-2019.

Corresponding Author:

Dr. Suhas G. Kumbhar,

Professor, Department of Paediatrics,

Bharati Vidyapeeth (Deemed to be University)

Medical College and Hospital,

Sangli, AIP Walness Wadi-416414, Maharashtra, India.

E-mail: suhasvidhya123@gmail.com

DOI: $10.14260 /$ jemds $/ 2019 / 375$
Up to $10 \%$ of all new cases of pneumonia progress to severe episodes and require hospitalization. Electrolyte disturbances have been described in a wide variety of acute infections including pneumonia which complicate the management and prognosis. $(4,5,6)$

Fluids and electrolytes are the primary pillars in the upkeep of body homeostasis. The most essential among electrolytes is sodium which is the abundant cation of the extracellular fluid(7). Hyponatremia is the most common electrolyte irregularity seen in the intensive care unit (ICU), with an occurrence as high as $30 \%$ in some reports. Hyponatremia is seen in various inflammatory diseases such as acute meningitis, acute lower respiratory tract infections, febrile convulsions, and Kawasaki disease in children.(1,6)

Hyponatremia is defined as a serum sodium level below $135 \mathrm{mmol} / \mathrm{L}$. The hyponatremia rate in inpatients is around $15 \%-30 \%$. Hyponatremia can be classified into three groups as mild (131-135 mmol/L), moderate (126-130 mmol/L) and severe $(\leq 125 \mathrm{mmol} / \mathrm{L})$. Mild hyponatremia is the most common type and usually produces no clinical findings. Severe hyponatremia is rare and has high morbidity and mortality rates if not treated. $(8,9)$ 
There are multiple factors contribute for the development of hyponatremia in lower respiratory tract infection. The various mechanisms are primary illness, impaired water secretion, syndrome of inappropriate antidiuretic hormone secretion (SIADH) $(5,10)$, use of hypotonic saline, redistribution of sodium and water and several drugs may contribute to the development of hyponatremia. The secretion of antidiuretic hormone is triggered by multiple osmotic stimuli like hyperglycaemia, hypovolemia, hypercapnia and pain. Sources of free water include hypotonic intravenous fluids, Ryle's tube feeding and humidified air in the ventilator circuit. These above factors are commonly seen in children who are admitted in paediatric intensive care unit suffering from acute lower respiratory tract infection(1,11).

Acute hyponatremia poses an immediate danger to the central nervous system. The rapid shift of fluid associated with this condition, frequently results in cerebral oedema. Administration of hypotonic maintenance fluids may worsen this oedema. $(4,9)$

This study has been taken to find out the occurrence of hyponatremia in children suffering from acute lower respiratory tract infection in hospital settings. In this study we are also going to compare the occurrence of hyponatremia in pneumonia group and other types of lower respiratory tract infection.

\section{METHODS}

This was a cross-sectional study carried out in paediatric intensive care unit (PICU) and Paediatric ward at tertiary hospital, Sangli. All children presenting with clinical symptoms suggestive of acute lower respiratory tract infections were evaluated in detail in hospital on IPD basis. Informed consent was taken. This study was approved by our institutional ethical committee (IEC).

After an informed consent (Parent or guardian) in children, a detailed history was taken, and physical examination was done. Information on socio-demographic variables including child's age, gender and also symptoms of acute lower respiratory tract infection, such as the presence and duration of fever associated with chills, cough, nasal discharge, breathlessness or fast breathing and abdominal pain was taken. To know the severity of acute lower respiratory tract infection history of symptoms like grunting, noisy respiration, chest pain was elaborated.

On general examination the child was examined for signs of respiratory distress like flaring of ala nasi, intercostal and subcostal retractions of chest. Detailed respiratory system examination was done in all patients in relation to inspection, palpation, percussion and auscultation.

Based on the history, general physical examination and systemic examination those children who fulfilled the criteria of acute lower respiratory tract infection mostly on the basis of latest integrated management of neonatal and childhood illness (IMNCI) guidelines were included in the study.

After clinical diagnosis, basic laboratory investigations like Total white blood cells (WBC), erythrocyte sedimentation rate (ESR), CRP, blood culture and chest x-ray were done in each patient. Depending upon the situation of the patient,
Chest USG or HRCT chest was done. Same venous sample collected in plain bulb on admission was sent to biochemistry laboratory for serum sodium level measurement. Serum sodium levels were measured using Patholyte-3 machine. Serum sodium levels were measured using a standardization of Sr Na-145.

Hyponatremia was defined as a serum sodium level below $135 \mathrm{mmol} / \mathrm{L}$. Hyponatremia was classified into three groups as mild (131-135 mmol/L), moderate (126-130 mmol/L), and severe $(\leq 125 \mathrm{mmol} / \mathrm{L}) .{ }^{(8)}$

Disease severity was assessed by respiratory distress, Sp02, presence of general danger signs as per integrated management of neonatal and childhood illness (IMNCI) guidelines, respiratory support needed and duration of hospitalisation.

The final type of acute lower respiratory tract infection was decided on the basis of history, physical examination and detailed investigations.

The frequency of hyponatremia was calculated in all study patients in relation to age, sex and expressed as percentage. The frequency of hyponatremia in pneumonia patients and other types of acute lower respiratory tract infection was estimated and compared.

All relevant data was filled in Microsoft excel sheet and analysis was done using SPSS Version 13. The comparison was done by $\mathrm{z}$ test. $\mathrm{P}$ value $<0.05$ was considered as significant.

\section{RESULTS}

85 cases of lower respiratory tract infection (8\%) of total 925 admissions during study period were included in the study. Out of the 85 cases of lower respiratory tract infection, 59 $(69.4 \%)$ had pneumonia, 16 (18.8\%) had bronchiolitis, 8 (9.4\%) had wheeze associated lower respiratory tract infection and $2(2.35 \%)$ presented with acute CROUP syndrome. The prevalence of hyponatremia in lower respiratory tract infection was found to be $42.3 \%$ in our study.

Hyponatremia was seen in $50 \%$ of lower respiratory tract infection below 1 year of age as compared to $30 \%$ seen between 3-5 years of age. Only $19.44 \%$ of hyponatremia cases were present between 13 months to 36 months of age. Males were twice more commonly affected than females with hyponatremia in acute lower respiratory tract infection.

Hyponatremia was found with highest percentage $(69.4 \%)$ in children suffering from pneumonia group. Out of 36 cases of hyponatremia associated with lower respiratory tract infection, 25 (69.4\%) had mild hyponatremia, 8 (22.2\%) had moderate hyponatremia and $3(8.3 \%)$ had severe hyponatremia.

Hyponatremia was found in all cases of very severe pneumonia i.e. $13(100 \%)$ as compared to $16(53.33 \%)$ in severe pneumonia group and only $3(18.75 \%)$ in pneumonia group, but there was no significant association found between severity of hyponatremia and pneumonia grading as the $\mathrm{p}$ value was $0.145(<0.05)$.

In our study, out of 36 cases of hyponatremia, $33(91.66 \%)$ were present in pneumonia group and only $3(8.33 \%)$ presented in non-pneumonia group. As $p$ value is 0.00 and $\mathrm{z}$ value is 3.817 , the above result was found to be statistically significant. 


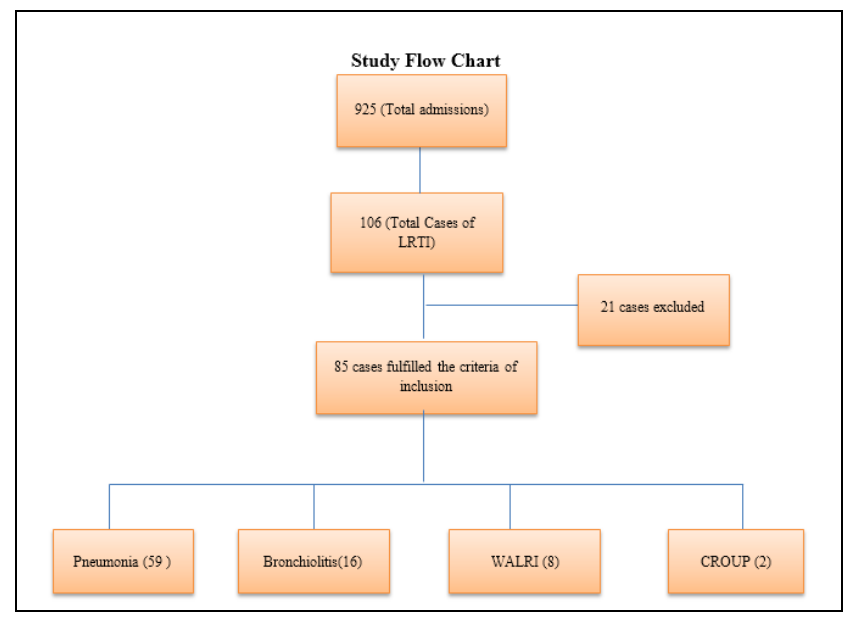

\begin{tabular}{|c|c|c|}
\hline Age & No. of Hyponatremia Cases (n=36) & Percentage \\
\hline 2 -12 Months & 18 & $50 \%$ \\
\hline 13-36 Months & 7 & $19.44 \%$ \\
\hline 37-60 Months & 11 & $30.55 \%$ \\
\hline \multicolumn{3}{|c|}{ Table 1. Age Distribution in Hyponatremia in Lower Respiratory } \\
Tract Infection Patients \\
\hline
\end{tabular}

\begin{tabular}{|c|c|c|}
\hline Total Cases & $\mathbf{8 5}$ & Percentage \\
\hline Pneumonia & 59 & $69.4 \%$ \\
\hline Bronchiolitis & 16 & $18.8 \%$ \\
\hline WALRI & 8 & $9.4 \%$ \\
\hline Acute Croup Syndrome & 2 & $2.35 \%$ \\
\hline \multicolumn{2}{|c|}{ Table 2. Different Lower Respiratory Tract Conditions } \\
\hline
\end{tabular}

\begin{tabular}{|c|c|c|c|}
\hline & $\begin{array}{c}\text { Mild } \\
\text { Hyponatremia } \\
(\mathbf{n = 2 1 )}\end{array}$ & $\begin{array}{c}\text { Moderate } \\
\text { Hyponatremia } \\
(\mathbf{n = 9 )}\end{array}$ & $\begin{array}{c}\text { Severe } \\
\text { Hyponatremia } \\
(\mathbf{n = 3 )}\end{array}$ \\
\hline Pneumonia & 4 & - & - \\
\hline Severe Pneumonia & 12 & 3 & 1 \\
\hline $\begin{array}{c}\text { Very Severe } \\
\text { Pneumonia }\end{array}$ & 5 & 6 & 2 \\
\hline
\end{tabular}

Table 3. Severity of Hyponatremia with Pneumonia Grading

Fisher exact test: $\mathrm{p}$ value $=0.1494$ There was no significant association was found between hyponatremia with pneumonia grading.

\begin{tabular}{|c|c|c|}
\hline LRT Conditions & $\begin{array}{c}\text { Hyponatremia } \\
(\mathbf{n}=\mathbf{3 6})\end{array}$ & Percentage \\
\hline Pneumonia (n=59) & 33 & $91.66 \%$ \\
\hline Non-Pneumonia Group (n=26) & 3 & $8.33 \%$ \\
\hline Table 4. Comparison of Hyponatremia in Pneumonia and Other \\
Lower Respiratory Tract Conditions \\
\hline \multicolumn{2}{|c|}{ The Z value is 3.817. The p value is 0.000. Result is significant at $\mathrm{p}<0.05$} \\
\hline
\end{tabular}

\section{DISCUSSION}

Hyponatremia is commonest electrolyte abnormality seen amongst hospitalized children with lower respiratory tract conditions(10). Presence of hyponatremia substantially increases the morbidity and mortality in children with lower respiratory tract conditions. $(1,11,12)$

Acute, severe hyponatremia that develops within 48 hours can result in acute cerebral oedema and various complications such as headache, lethargy, seizures, and cardiac arrest due to brain stem herniation. Children are more vulnerable than adults to above complications because the brain/intracranial volume ratio is higher in children than in adults. $(13,14,15)$

Recent evidence suggests that even mild chronic hyponatremia can be related to subtle neurologic defects, such as impairments in balance and attention that can increase the incidence of falls.(15)
In our study out of the 85 cases of lower respiratory tract infection, 59 (69.4\%) had pneumonia, 16 (18.8\%) had bronchiolitis, 8 (9.4\%) had wheeze associated lower respiratory tract infection and $2(2.35 \%)$ presented with acute CROUP syndrome. The prevalence of hyponatremia in lower respiratory tract infection was found to be $42.3 \%$. Similar results were seen in study done by Chaitra K M et al. where out of total 91 cases of lower respiratory tract infection, $72(79.1 \%)$ had pneumonia, 6 (6.5\%) had bronchiolitis, $8(8.7 \%)$ had wheeze associated lower respiratory tract infection and 5 (5.4\%) presented with empyema. Prevalence of hyponatremia was found to be $47.7 \%$, which was similar to the results observed in our study(2).

The most probable cause of hyponatremia is thought to be the non-osmotic release of antidiuretic hormone (ADH) as a result of various clinical conditions, such as fever, hypovolemia, and respiratory tract infections. $(16,17,5)$

Hyponatremia occurring in children with pneumonia comprises part of the syndrome of inappropriate antidiuretic hormone secretion (SIADH). Antidiuretic hormone is generally secreted by the pituitary gland in response to high plasma osmolality (High serum sodium concentration) however in various clinical conditions, including fever, hypoxia, hypercarbia, pain, nausea, and vomiting, nonosmotic stimulation of antidiuretic hormone secretion can lead to hyponatremia(10).

Among patients with respiratory tract infections, pneumonia and bronchiolitis are most commonly associated with hyponatremia.(17,18) However, the incidence of hyponatremia according to the etiological involvement of various microorganisms has not yet been studied.

In our study hyponatremia was seen in $50 \%$ of lower respiratory tract infection below 1 year of age as compared to $30 \%$ seen between 3-5 years of age. Only $19.44 \%$ of hyponatremia cases were present between 13 months to 36 months of age. The results obtained in our study were found to be comparable with the studies done by Chaitra $\mathrm{K} \mathrm{M}$ et al. In her study hyponatremia was seen in $63 \%$ of lower respiratory tract infection below 1 year of age, $24 \%$ between the age group of 13 months to 36 months and $5 \%$ between 3 5 years of age. Similar results were found in the study done by Sunil Mhaske et al. were hyponatremia was seen in $58.8 \%$ cases of lower respiratory tract infection below 1 year of age $\& 41.9 \%$ between the age of $1-5$ years of age ${ }^{(4)}$.

In our study males were twice more commonly affected than females with hyponatremia in acute lower respiratory tract infection. Similar results were found in the study done by Chaitra $\mathrm{K} \mathrm{M}$ et al. where $59 \%$ males were affected as compared to $41 \%$ in females(2). In study done Sunil Mhaske et al. $51.7 \%$ males were affected \& $48.3 \%$ females were affected. Similarly in study done by Yilmaz et al $61 \%$ of males were affected as compared to $39 \%$ seen in females $(8)$.

The present study showed, out of 36 cases of hyponatremia associated with lower respiratory tract infection, 25 (69.4\%) had mild hyponatremia, 8 (22.2\%) had moderate hyponatremia and $3(8.3 \%)$ had severe hyponatremia. Similar results were seen in the study done by Chaitra K M et al. where out of 41 cases of hyponatremia associated with lower respiratory tract infection, 29 (70.7\%) had mild hyponatremia, 9 (21.9\%) had moderate hyponatremia \& 3 (7.3\%) had severe hyponatremia(2). 
Similarly, in the study done by Channawar K S out of the 54 cases of hyponatremia $10 \quad(18.5 \%)$ had moderate hyponatremia and $3(5.5 \%)$ had severe hyponatremia(9). In study done by Sakellaropoulou A et al. out of 54 cases, 18 (33.3\%) cases had mild hyponatremia \& 1 (1.9\%) had moderate hyponatremia(11).

In our study, hyponatremia was found with highest percentage $(69.4 \%)$ in children suffering from pneumonia group. Hyponatremia was found in all cases of very severe pneumonia i.e. $13(100 \%)$ as compared to $16(53.33 \%)$ in severe pneumonia group and only $3(18.75 \%)$ in pneumonia group, but there was no significant association found between severity of hyponatremia and pneumonia grading as the $\mathrm{p}$ value was $0.145(<0.05)$. In study done by Chaitra K. M et $\mathrm{al}^{(2)}$ pneumonia was the commonest lower respiratory tract condition seen in $79.12 \%$, in which prevalence of hyponatremia was seen in $100 \%$ in all empyema cases and $46.7 \%$ in bronchopneumonia cases. Above results were comparable with our study.

In our study, out of 36 cases of hyponatremia, 33 (91.66\%) were present in pneumonia group and only $3(8.33 \%)$ presented in non-pneumonia group. As $p$ value is 0.00 and $\mathrm{z}$ value is 3.817 , the above result was found to be statistically significant. Similar results were seen in the study done by Chaitra K.M. et al(2) were out of 41 cases of hyponatremia, 34 (82.9\%) were present in pneumonia group and only 7 (17.07) presented in non-pneumonia group.

\section{CONCLUSIONS}

The prevalence of hyponatremia in paediatric patients with lower respiratory tract infection was found to be $42.3 \%$. Hyponatremia was more common in pneumonia group (91.66\%) as compared to the non-pneumonia group (8.33\%). Severe hyponatremia was seen more commonly with increase in severity of pneumonia; so, diagnosing hyponatremia in early stage and correcting by appropriate interventions plays an important role in outcome of children suffering from pneumonia(3,19).

\section{REFERENCES}

[1] Nasser AH, Abdullah AA, Saad AA, et al. Hyponatremia in children with acute lower respiratory tract infections: overview. International Journal of Healthcare Sciences 2016;4(2):4859. www.researchpublish.com.

[2] Chaitra KM, Kumar MN, Reddy SG. Hyponatremia in lower respiratory tract infections. International journal of contemporary Pediatrics 2016;3(2):3814. http://www.ijpediatrics.com.

[3] Don M, Fasoli L, Paldanius M, et al. Aetiology of community-acquired pneumonia: serologic results of a paediatric survey. Scand J Infect Dis 2005;37(1112):806-12.
[4] Mhaske S, Bulsara L, Kadam V. Correlation of hyponatremia in children presenting with acute lower respiratory infection in a tertiary care hospital tract. The Antiseptic 2016;113(6):24-7.

[5] Singhi S, Dhawan A. Frequency and significance of electrolyte abnormalities in pneumonia. Indian Pediatr 1992;29(6):735-40.

[6] Don M, Paldanius M, Fasoli L, et al. Simkania negevensis and pneumonia in children. Pediatr Infec Dis J 2006;25(5):470-1.

[7] Kliegman RM, Stanton BF, Joseph W, et al. Nelson Textbook of Pediatrics. 20 $0^{\text {th }}$ edn. Philadelphia: Elsevier 2016.

[8] Yilmaz Y, Candar T, Kara F, et al. Serum sodium levels in children with lung infections. J Pediatr Inf 2016;10:10-3. www.jpediatrinf.org.

[9] Channawar KS, Deshmukh N, Prasad VSV. Correlation of hyponatremia in children with lower respiratory tract infection - an institutional observational study. J Evolution Med Dent Sci 2016;5(88):6533-5.

[10] Dhawan A, Narang A, Singhi S. Hyponatremia and the inappropriate ADH syndrome in pneumonia. Ann Trop Paediatr 1992;12(4):455-62.

[11] Sakellaropoulou A, Hatzistilianou M, Eboriadou M, et al. Hyponatremia in cases of children with pneumonia. Arch Med Sci 2010;6(4):578-83.

[12] Nair V, Niederman MS, Masani N, et al. Hyponatremia in community-acquired pneumonia. Am J Nephrol 2007;27(2):184-90.

[13] Moritz ML, Ayus JC. Preventing neurological complications from dysnatremias in children. Pediatr Nephrol 2005;20(12):1687-700.

[14] Moritz ML, Ayus JC. New aspects in the pathogenesis, prevention and treatment of hyponatremic encephalopathy in children. Pediatr Nephrol 2010;25(7):1225-38.

[15] Moritz ML, Ayus JC. Maintenance intravenous fluids in acutely ill patients. N Engl J Med 2016;374(3):290-1.

[16] Hasegawa H, Okubo S, Ikezumi Y, et al. Hyponatremia due to an excess of arginine vasopressin is common in children with febrile disease. Pediatr Nephrol 2009;24(3):507-11.

[17] Dhawan A, Narang A, Singhi S. Hyponatraemia and the inappropriate ADH syndrome in pneumonia. Ann Trop Paediatr 1992;12(4):455-62.

[18] Hanna S, Tibby SM, Durward A, et al. Incidence of hyponatraemia and hyponatraemic seizures in severe respiratory syncytial virus bronchiolitis. Acta Paediatr 2003;92(4):430-4.

[19] Stormont JM, Waterhouse C. Severe hyponatremia associated with pneumonia. Metabolism 1962;11:1181-6. 